\title{
Non-contrast second harmonic imaging improves interobserver agreement and accuracy of dobutamine stress echocardiography in patients with impaired image quality
}

\author{
A Franke, R Hoffmann, H P Kühl, W Lepper, O A Breithardt, M Schormann, P Hanrath
}

\begin{abstract}
Objective-To examine the influence of second harmonic imaging during dobutamine echocardiography on regional endocardial visibility, interobserver agreement in the interpretation of wall motion abnormalities, and diagnostic accuracy in patients with reduced image quality.

Design-Blinded comparison.

Setting-Tertiary care centre.

Patients-103 consecutive patients with suspected coronary artery disease and impaired transthoracic image quality ( $\geqslant 2$ segments with poor endocardial delineation).

Methods-Fundamental and second harmonic imaging were performed at each stage of a dobutamine stress echocardiography. Coronary angiography was undertaken within three weeks of dobutamine echocardiography in 75 patients.

Main outcome measures-Evaluation of regional endocardial visibility (scoring from $0=$ poor to $2=$ good) and of segmental wall motion abnormalities for both modalities separately. A second blinded examiner analysed 70 studies to determine interobserver agreement.

Results-Mean (SD) visibility score for all segments was $1.2(0.4)$ using fundamental imaging and $1.7(0.2)$ using second harmonic imaging at rest $(\mathrm{p}<0.001)$, and $1.1(0.4) v 1.6(0.3)$, respectively, at peak dobutamine dose $(\mathrm{p}<0.001)$. The average number of segments with poor endocardial visibility was lower for second harmonic than for fundamental imaging $(0.6(1.1) v$ $3.8(2.6)$ at rest, $\mathrm{p}<0.001 ; 0.9$ (1.3) $v 4.3(2.9)$ at peak dose, $\mathrm{p}<0.001)$. Improvement was most pronounced in all lateral and anterior segments. The $\kappa$ value for identical study interpretation increased from 0.40 to 0.69 ( $\mathrm{p}<0.05)$. Sensitivity for the diagnosis of coronary artery disease was $64 \%$ using fundamental imaging versus $92 \%$ using harmonic imaging $(\mathrm{p}<0.001)$, while specificity remained unchanged at $75 \%$ for both imaging modalities.

Conclusions-Second harmonic imaging enhances endocardial visibility during dobutamine echocardiography. Consequently, interobserver agreement on stress echocardiography interpretation and diagnostic accuracy are significantly improved compared to fundamental imaging. Thus, in difficult to image patients, dobutamine echocardiography should be performed using second harmonic imaging.

(Heart 2000;83:133-140)
\end{abstract}

Keywords: coronary artery disease; dobutamine stress echocardiography; second harmonic imaging; interobserver agreement

Dobutamine stress echocardiography has gained widespread use to detect coronary artery disease and to evaluate the functional relevance of angiographically proven coronary lesions. Several previous studies have shown its high diagnostic accuracy. ${ }^{1-5}$ However, reading of dobutamine stress echocardiography is dependent on subjective interpretation of visual information, a process which has been shown to be extremely sensitive to the experience of the echo reader. ${ }^{6}$ Furthermore, there may be considerable differences in the interpretation of dobutamine stress echocardiograms, even among experienced readers. ${ }^{7}$ Image quality has a significant influence on the interpretation of dobutamine stress echocardiography studies. While low image quality results in a high degree of uncertainty as to the myocardial contraction pattern, high image quality narrows the interpretive range. Thus, any imaging modality that improves echocardiographic image quality should have an impact on interobserver agreement and diagnostic accuracy of dobutamine stress echocardiography.

Recently, several reports recognised the benefit of second harmonic imaging for the improvement of endocardial delineation, even without the use of contrast agents. ${ }^{8-10}$ The technique of second harmonic imaging uses broadband transducers which receive double the transmitted frequency. Harmonic imaging was initially developed to intensify the ultrasound signal during left heart contrast echocardiography, based on the fact that most contrast agents emit harmonic frequencies when hit by ultrasound energy. ${ }^{11}{ }^{12}$ Whether the benefits of non-contrast second harmonic imaging can be extended to dobutamine stress echocardiography has not been evaluated yet.

Thus, our study aimed to examine the influence of second harmonic imaging on: the regional endocardial visibility during dobutamine stress echocardiography; the 
interobserver agreement of dobutamine stress echocardiography interpretation; and the sensitivity, specificity, and accuracy of dobutamine stress echocardiography compared to conventional fundamental imaging in patients with impaired image quality.

\section{Methods}

PATIENT CHARACTERISTICS

The study included 103 consecutive patients with impaired transthoracic image quality during baseline echocardiography using fundamental imaging. Impaired image quality was defined as two or more segments of the $16 \mathrm{seg}$ ment model of the American Society of Echocardiography ${ }^{13}$ showing poor or no endocardial delineation, as judged by an experienced echocardiographer. Dobutamine stress echocardiography was performed for suspected coronary artery disease or to evaluate the functional relevance of known coronary stenoses. All patients gave their informed consent. The usual exclusion criteria for stress testing were applied. ${ }^{14}$ Antianginal treatment before examination included nitrates in 79 patients, calcium channel antagonists in 70 patients, and $\beta$ adrenergic blocking agents in 58 patients. Previous myocardial revascularisation had been performed in 23 patients (13 with coronary angioplasty, 10 with coronary bypass grafting), and 27 patients had a history of previous myocardial infarction. The baseline echocardiogram showed regional wall motion abnormalities in 39 patients. Circumstances which might result in impaired transthoracic image quality were seen in 82 patients $(80 \%)$ : obesity with a body mass index $>25 \mathrm{~kg} / \mathrm{m}^{2}$ was present in 67 patients $(65 \%)$; chronic obstructive lung disease in 10 patients (10\%); and previous thoracic surgery in 18 patients $(17 \%)$.

Coronary angiography was performed within three weeks before or after dobutamine stress echocardiography in 75 patients, who either had objective criteria of myocardial ischaemia (exercise ECG, nuclear imaging, or stress echocardiography, 61 patients) or complained of typical anginal symptoms in the absence of objective criteria for myocardial ischaemia (14 patients). Relevant coronary artery disease was excluded in 15 of these patients $(20 \%)$; the remaining 60 patients had coronary artery disease, with one vessel disease in 39 patients (52\%), two vessel disease in 10 patients $(13 \%)$, and three vessel disease in 11 patients $(15 \%)$. The demographic and clinical data of the patients are given in table 1 .

DOBUTAMINE STRESS ECHOCARDIOGRAPHY

Dobutamine infusion was started at $10 \mu \mathrm{g} / \mathrm{kg} /$ min and increased in $10 \mu \mathrm{g}$ increments every three minutes up to $40 \mu \mathrm{g} / \mathrm{kg} / \mathrm{min}$. If heart rate response was inadequate and below $85 \%$ of maximal age predicted heart rate, $0.25 \mathrm{mg}$ atropine was administered intravenously every following minute up to a maximum of $1 \mathrm{mg}$. ${ }^{15}$ A 12 lead ECG was monitored continuously and blood pressure was measured by cuff method every two minutes during and up to 10 minutes after dobutamine stress echocardio-
Table 1 Demographic, clinical, and angiographic data of the 103 study patients

\begin{tabular}{ll}
\hline Mean (SD) age (years) & $59(11)$ \\
Male/female & $82 / 21$ \\
Wall motion abnormalities at baseline & \\
$\quad$ echocardiogram & 39 \\
Mean (SD) body mass index $\left(\mathrm{kg} / \mathrm{m}^{2}\right)$ & $26.2(3.5)$ \\
Coronary angiography performed & 75 \\
Coronary artery disease excluded & 15 \\
One vessel disease & 39 \\
Two vessel disease & 10 \\
Three vessel disease & 11 \\
\hline
\end{tabular}

Table 2 Clinical parameters (mean (SD)) at peak dobutamine dose during dobutamine stress dobutamine dose
echocardiography

Peak dobutamine dose $(\mu \mathrm{g} / \mathrm{kg} / \mathrm{min})$ Maximal heart rate $(1 / \mathrm{min})$ $34.4(7.4)$ Maximal systolic blood pressure $(\mathrm{mm} \mathrm{Hg})$ Maximal diastolic blood pressure (mm Hg) $90.5(14.0)$ Rate-pressure product ( $\mathrm{mm} \mathrm{Hg} / \mathrm{min}$ )

graphy. The clinical parameters at peak dose are given in table 2 .

Loops of one heart cycle were stored at four levels during the dobutamine stress echocardiography (at baseline, low dose after three minutes of $10 \mu \mathrm{g} / \mathrm{kg} / \mathrm{min}$ dobutamine, peak dose, and during recovery). Four standard views were acquired at all four levels: parasternal long and short axis as well as apical four and two chamber view. For the acquisition of stress echocardiography studies either an Acuson Sequoia 256 system (Acuson, Mountainview, California, USA) with a $3.5 \mathrm{MHz}$ fundamental transducer and $1.75 / 3.5 \mathrm{MHz}$ second harmonic imaging capability or a Hewlett Packard Sonos 5500 (Hewlett Packard, Andover, Massachusetts, USA) with a $4 \mathrm{MHz}$ fundamental transducer $(2.1 / 4.2 \mathrm{MHz}$ for second harmonic imaging) was used. All studies were stored in digital format on magnetooptical disks. Both modalities (fundamental imaging and second harmonic imaging) were performed at every step (baseline, low dose, peak, and recovery) immediately one after another, resulting in 32 loops per examination and per patient.

The end points for the dobutamine infusion were: attainment of $85 \%$ of the age predicted maximal heart rate; systolic or diastolic blood pressure $>240 \mathrm{~mm} \mathrm{Hg}$ or $>120 \mathrm{~mm} \mathrm{Hg}$, respectively; a decrease in blood pressure $>20 \mathrm{~mm} \mathrm{Hg}$; serious arrhythmia; and development of a new wall motion abnormality or angina. Seventy two of the 103 patients $(70 \%)$ reached $>85 \%$ of the age predicted maximal heart rate. In 14 patients (14\%) the stress test was stopped prematurely because of hypo- or hypertension $(\mathrm{n}=4)$ or because of arrhythmia $(\mathrm{n}=10)$, including two patients with a nonsustained ventricular tachycardia. Sixteen patients $(16 \%)$ developed wall motion abnormalities or angina before reaching target heart rate, and one patient had significant temporary ST elevation. There were no other relevant complications.

INTERPRETATION OF DOBUTAMINE STRESS ECHOCARDIOGRAPHY STUDIES

All stress echocardiograms were evaluated by an experienced blinded observer separately for 


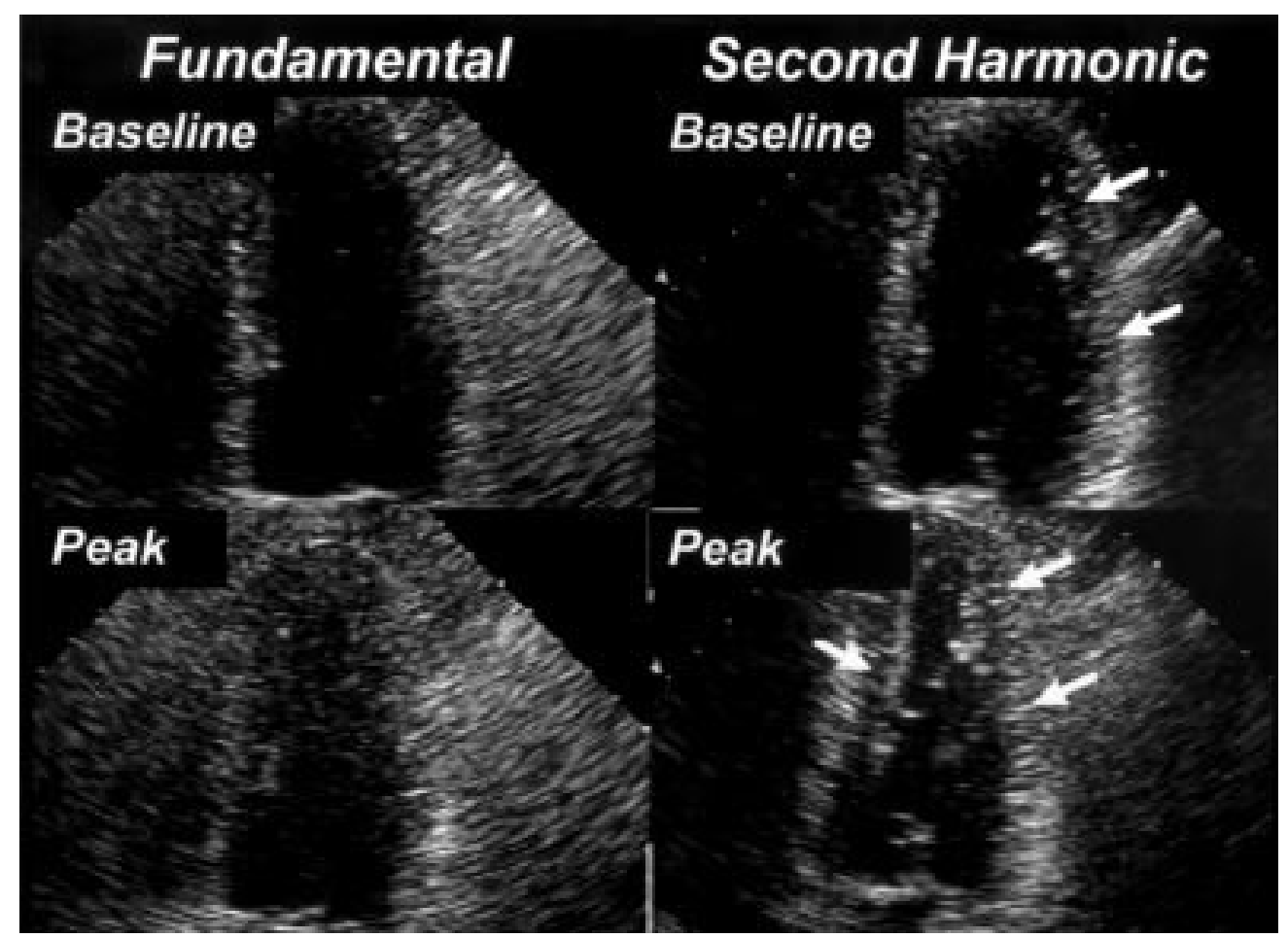

Figure 1 Effect of harmonic imaging on the endocardial visibility. Systolic stop frame images of the apical four chamber view at baseline (upper panels) and at peak dobutamine dose (lower panels). Fundamental images on the left, second harmonic images on the right. Note the improved endocardial delineation in the lateral segments at the baseline study and in both the septal and lateral segments during peak stress (arrows).

fundamental and second harmonic imaging with regard to endocardial visibility and wall motion abnormalities. The 16 segment model of the American Society of Echocardiography ${ }^{13}$ was used for interpretation.

\section{Evaluation of endocardial visibility}

Endocardial visibility of each segment was evaluated separately for both imaging modalities at rest and peak dobutamine dose. A scoring system was used from $0=$ barely or not

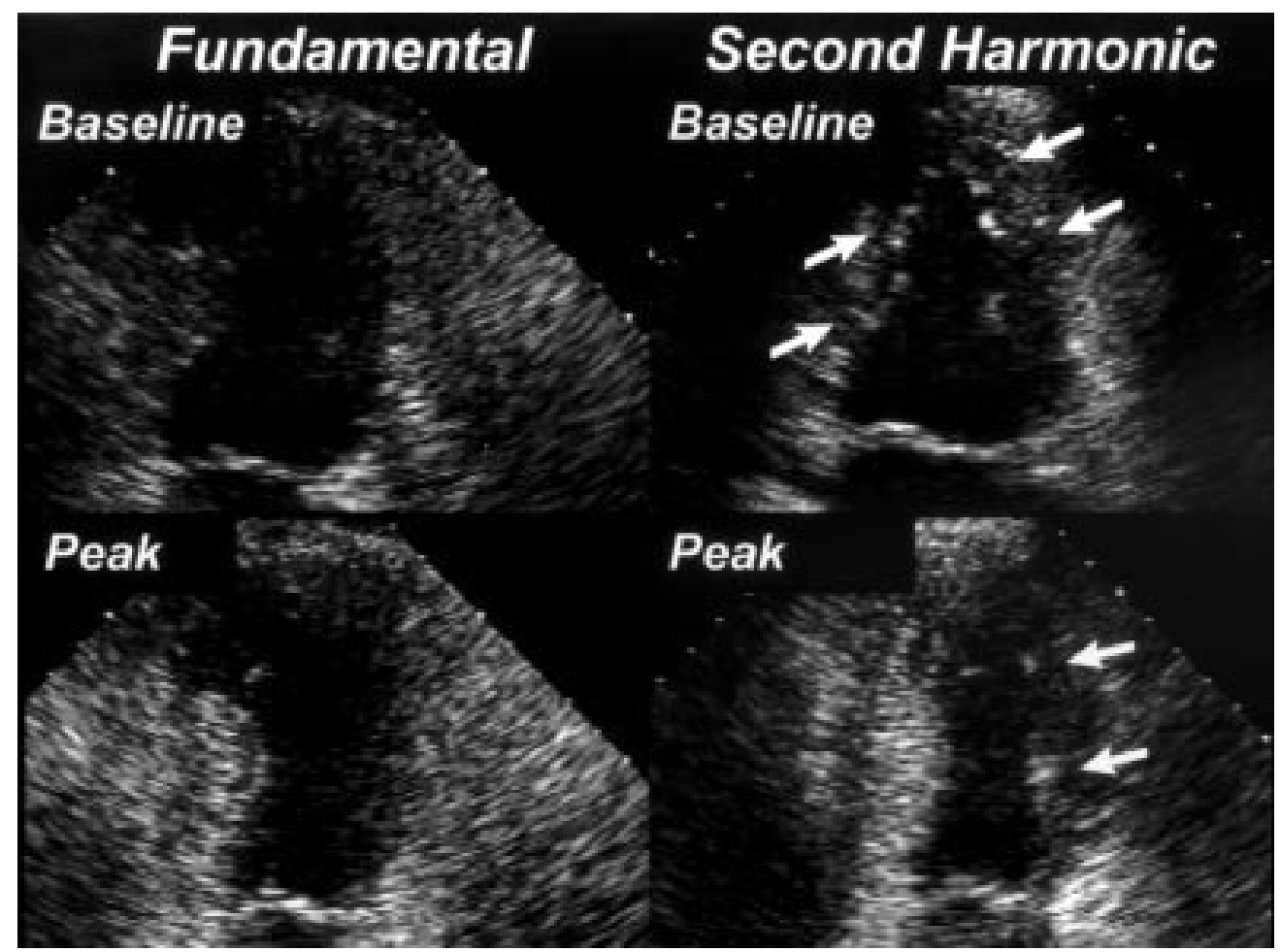

Figure 2 Effect of harmonic imaging on the endocardial visibility. Stop frame images of the apical two chamber view. Same orientation as in fig 1. The better endocardial delineation at peak stress is most pronounced in the anterior segments (arrows). 
Table 3 Mean (SD) visibility scores with both imaging modalities

\begin{tabular}{llll}
\hline & $\begin{array}{l}\text { Fundamental } \\
\text { imaging }\end{array}$ & $\begin{array}{l}\text { Second } \\
\text { harmonic } \\
\text { imaging }\end{array}$ & Significance \\
\hline Baseline & $1.2(0.4)$ & $1.7(0.2)$ & $\mathrm{p}<0.001$ \\
Peak dobutamine dose & $1.1(0.4)$ & $1.6(0.3)$ & $\mathrm{p}<0.001$ \\
\hline
\end{tabular}

visible endocardium, to $1=$ moderately or partly visible endocardium, to $2=$ good visible endocardium. For myocardial segments visible in more than one view the higher visibility score was used for further analysis.

Assessment of regional wall motion

Wall motion was evaluated using the conventional scoring: $0=$ not evaluable; $1=$ normokinetic; 2 =hypokinetic; 3 = akinetic; $4=$ dyskinetic. $^{14}$ The different stages of the examination were compared side by side using a quad screen display. Test positivity was defined as new or worsening wall motion abnormalities in at least one segment.

Interobserver agreement.

A subgroup of 70 patients was evaluated by a second blinded observer. Interobserver agreement on test positivity or negativity of dobutamine stress echo interpretation was analysed. Furthermore, for each of the 16 segments the results of both observers' wall motion analyses were compared separately at baseline and peak dobutamine dose using fundamental and second harmonic imaging.

QUANTITATIVE CORONARY ANGIOGRAPHY

Coronary angiograms were stored digitally (ACOM, Siemens, Erlangen, Germany) and the severity of coronary stenoses was analysed quantitatively using a commercially available software (QuantCor, CASS II, Siemens). A diameter stenosis of $\geqslant 50 \%$ from the worst view was considered significant to define a vessel as diseased.

STATISTICAL ANALYSIS

All continuous variables are expressed as mean (SD). Comparison between different modalities was performed using paired and unpaired Student's $t$ tests, Pearson's linear correlation

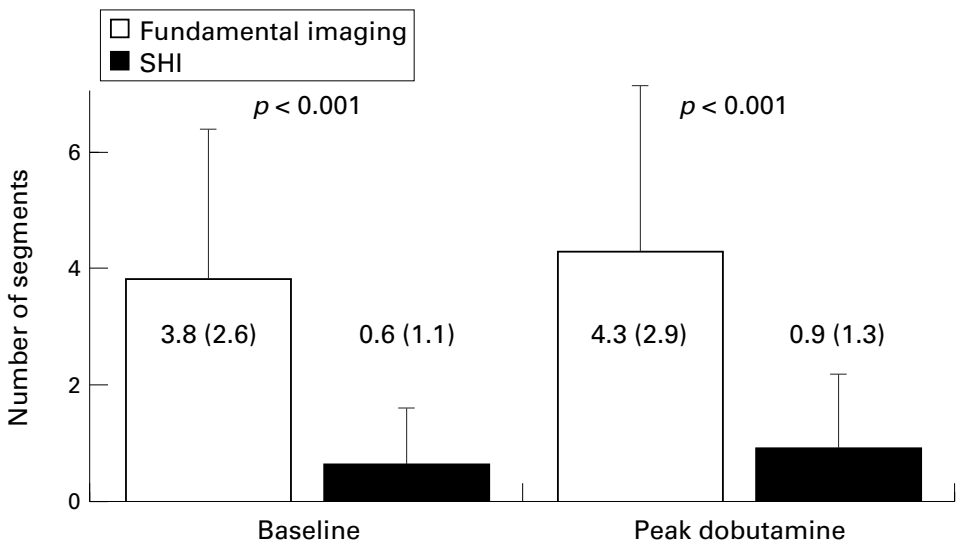

Figure 3 Average number of segments per patient with a visibility score of 0 at baseline (left) and at peak dobutamine dose (right). The number of segments with poor endocardial delineation with fundamental imaging decreases significantly using second harmonic imaging (SHI). This effect was present both at baseline and at peak dobutamine dose. and analysis of non-linear inverse relation for continuous variables, and $\chi^{2}$ test or Spearman's rank test for categorical variables as appropriate. A value of $\mathrm{p}<0.05$ was considered significant.

The Cohen- $\kappa$ coefficient for interobserver agreement was calculated as the difference between observed agreement and expected agreement by chance only divided by the maximal difference between observed and expected agreement. ${ }^{16} \mathrm{~A}$ value of 1.0 means perfect agreement of both observers in all cases; a value of 0 means that identical results are only caused by chance.

\section{Results}

ENDOCARDIAL VISIBILITY

The use of second harmonic imaging improved the endocardial delineation because of a stronger signal of the endocardium and a higher contrast between myocardium and left ventricular cavity (figs 1 and 2). The mean visibility score at rest for all segments of all 103 patients was $1.2(0.4)$ using fundamental imaging and $1.7(0.2)$ using second harmonic imaging $(p<0.001)$. At peak dobutamine dose the mean visibility score improved to a similar extent from $1.1(0.4)$ to $1.6(0.3)$ using second harmonic imaging $(\mathrm{p}<0.001)$ (table 3$)$. The number of non-evaluable segments per patient with a visibility score of 0 decreased significantly using second harmonic imaging compared to the fundamental technique $(0.6$ (1.1) $v 3.8(2.6)$ segments per patient at rest, $\mathrm{p}<0.001 ; 0.9$ (1.3) $v 4.3$ (2.9) segments per patient at peak dobutamine dose, $\mathrm{p}<0.001$ ) (fig 3).

Analysis of the regional distribution of improved visibility revealed that the benefit of second harmonic imaging was most pronounced in the middle segments of the parasternal long axis view, the anterior and lateral segments of the parasternal short axis, the lateral segments of the four chamber view, and the anterior segments of the two chamber view (figs 4 and 5). In these segments more than half of the patients revealed an improvement of at least one score point in the endocardial visibility.

The improved image quality using second harmonic imaging had a significant impact on the interpretation of dobutamine stress echocardiography. Using fundamental imaging, 11 of the 103 dobutamine stress echocardiography studies $(11 \%)$ were non-diagnostic, defined as poor endocardial delineation of all segments of at least one coronary perfusion bed. All of these 11 stress echocardiograms became diagnostic by the use of second harmonic imaging. The interpretation of 32 dobutamine stress echocardiography studies (31\%) changed using second harmonic imaging because of the better endocardial delineation. In 29 patients (28\%) previously undetected wall motion abnormalities could be demonstrated, and in three patients a wall motion abnormality suspected with fundamental imaging could not be confirmed using second harmonic imaging $(3 \%)$. 


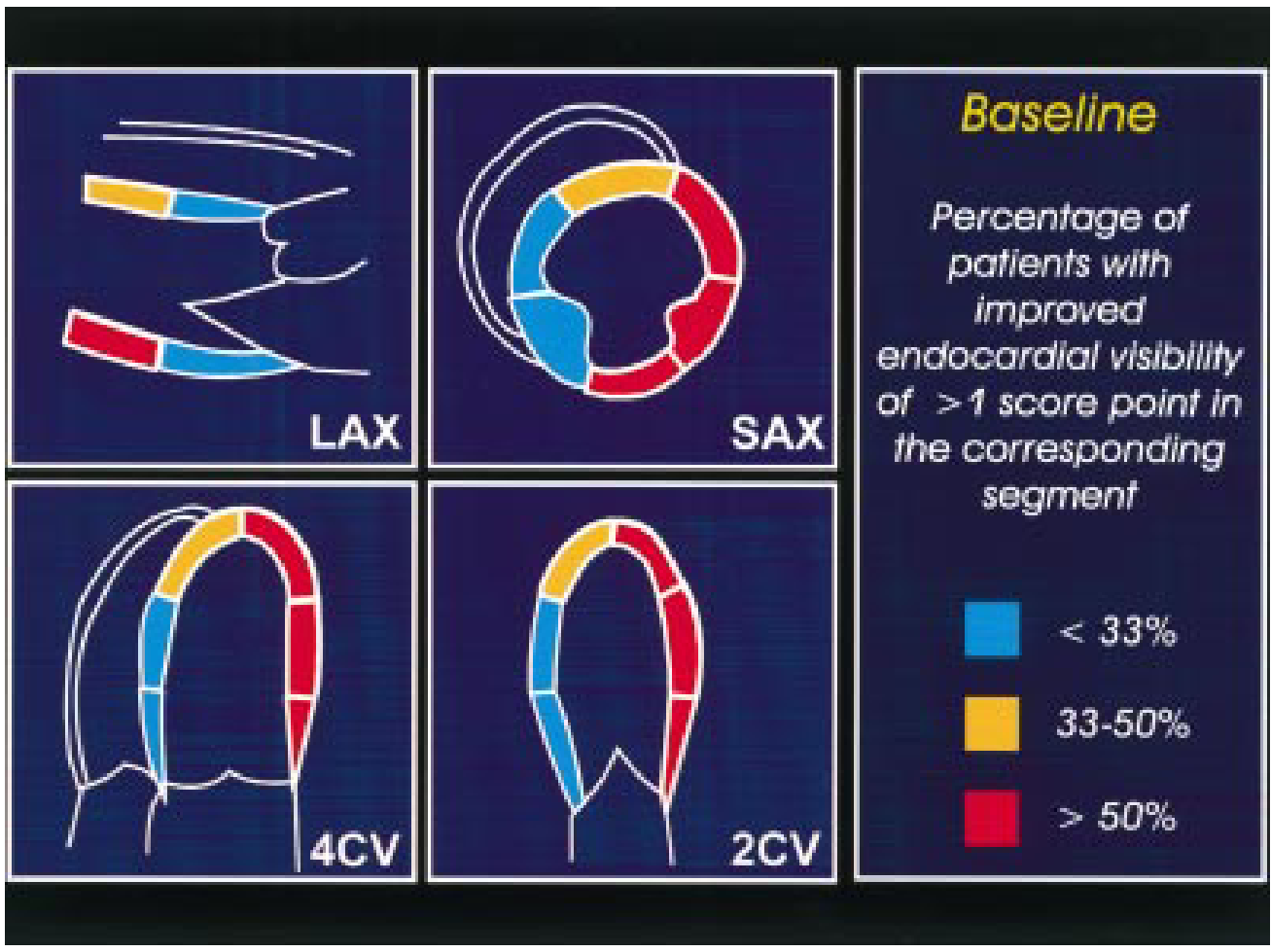

Figure 4 Analysis of the regional distribution of improved visibility at rest. The schematic drawing shows the four different image planes with all 16 segments. Segments where more than a third of the patients had an improvement of at least one score point using second harmonic imaging instead of fundamental imaging are shown in yellow. Segments where more than $50 \%$ of the patients had a better image quality are shown in red. Note that the benefit of second harmonic imaging is most pronounced in all lateral and anterior segments, where more than half of the patients improved. LAX, parasternal long axis, $S A X$, parasternal short axis; $4 C V$, apical four chamber view; $2 C V$, apical two chamber view.

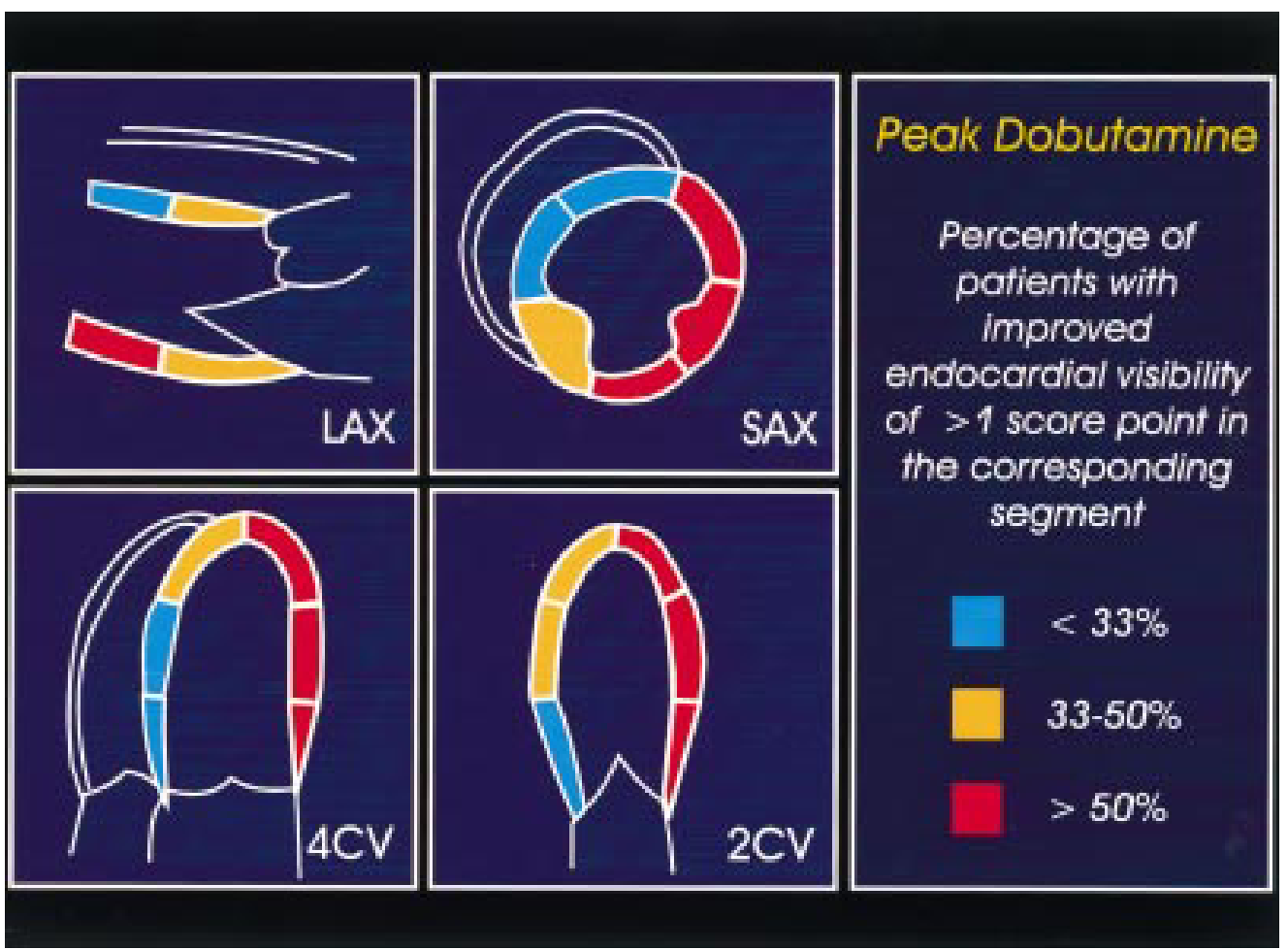

Figure 5 Regional distribution of improved visibility at peak dobutamine dose. The schematic drawing shows the four different image planes with all 16 segments. Segments where more than a third of the patients had an improvement of at least one score point using second harmonic imaging instead of fundamental imaging are shown in yellow. Segments where more than $50 \%$ of the patients had a better image quality are shown in red. Note that the benefit of second harmonic imaging is most pronounced in all lateral and anterior segments, where more than half of the patients improved. LAX, parasternal long axis; $S A X$, parasternal short axis; $4 C V$, apical four chamber view; $2 C V$, apical two chamber view. 
Table 4 Sensitivity, specificity, predictive values, and accuracy of dobutamine stress echocardiography with both imaging modalities for the detection of significant coronary artery disease

\begin{tabular}{llll}
\hline & $\begin{array}{l}\text { Fundamental } \\
\text { imaging }\end{array}$ & $\begin{array}{l}\text { Second } \\
\text { harmonic } \\
\text { imaging }\end{array}$ & Significance \\
\hline Sensitivity & $64 \%$ & $92 \%$ & $\mathrm{p}<0.001$ \\
Specificity & $75 \%$ & $75 \%$ & $\mathrm{NS}$ \\
Accuracy & $65 \%$ & $89 \%$ & $\mathrm{p}<0.001$ \\
\hline
\end{tabular}

INTEROBSERVER AGREEMENT

Analysis of the interobserver agreement in a subgroup of 70 patients showed a significant increase in the percentage of identically interpreted dobutamine stress echocardiography studies from $70 \%$ with fundamental imaging to $84 \%$ with second harmonic imaging ( $p<0.05)$. The $\kappa$ value for identical interpretation of a stress echocardiography study increased significantly from 0.40 to 0.69 ( $\mathrm{p}<0.05$ ). Similarly, there was agreement in the interpretation of single myocardial segments in 59\% with fundamental imaging and in $80 \%$ with second harmonic imaging. The corresponding $\kappa$ value increased from 0.49 to 0.73 using second harmonic imaging $(\mathrm{p}<0.001)$. The regional distribution of the improved agreement was similar to the distribution of improved endocardial visibility, and the benefit of second harmonic imaging was most pronounced in the lateral and anterior segments.

SENSITIVITY, SPECIFICITY, ACCURACY

The sensitivity of dobutamine stress echocardiography in the diagnosis of significant coronary artery disease was $64 \%$ with fundamental imaging and $92 \%$ with second harmonic imaging $(\mathrm{p}<0.001)$. Specificity was $75 \%$ for both imaging modalities (NS) (table 4). The accuracy increased from $65 \%$ to $89 \%$ $(\mathrm{p}<0.001)$.

\section{Discussion}

Despite the continuous evolution of imaging technology, the application of dobutamine stress echocardiography in clinical practice has been limited by impaired image quality at rest and even more so during stress. ${ }^{5}{ }^{17}$ Impaired image quality results in a reduced feasibility, a lower diagnostic accuracy, a lower interobserver agreement, and a greater level of insecurity about test results. ${ }^{7}$ Several new imaging modalities have been suggested for stress echocardiographic applications, including colour coded wall motion analysis and tissue velocity imaging. ${ }^{18} 19$ The proposed technologies have been aimed at improving image quality, thus lowering the degree of uncertainty and subjectivity involved in the interpretation of stress echocardiograms. Although these imaging modalities work in patients with already good images, they are rather disappointing in patients with poor acoustic windows.

Other reports showed a beneficial effect of left heart contrast agents, such as sonicated albumin, for improved endocardial border definition during dobutamine stress echocardiography with fundamental transducer technique..$^{20-22}$ However, these studies depend on the additional and sometimes cumbersome application of contrast agents and are limited by the possible attenuation where bolus injections are used.

Transoesophageal echocardiography in combination with transoesophageal atrial pacing or dobutamine stress has been suggested as an alternative approach in patients with impaired transthoracic image quality. ${ }^{23-25}$ In contrast to the previously mentioned techniques, image quality was especially improved in patients with poor transthoracic echo window. Because of limited patient tolerance, this approach has not gained wide clinical acceptance.

Several recent studies showed the benefit of non-contrast second harmonic imaging for the improvement of endocardial delineation, which was most pronounced in patients with impaired conventional transthoracic image quality. Most of these studies described the improvement of image quality using second harmonic imaging under resting conditions. ${ }^{8} 26$ However, the image quality at peak stress using the fundamental technique is especially sensitive to negative factors such as tachypnoea, excessive heart motion, and the need for rapid image acquisition, which impairs the diagnostic yield of the method. Therefore, this study was designed to assess the impact of harmonic imaging on the diagnostic accuracy of stress echocardiography.

IMAGE QUALITY USING SECOND HARMONIC IMAGING

The present study shows the benefit of harmonic imaging in dobutamine stress echocardiography without using left heart contrast agents. Second harmonic imaging significantly improved the endocardial delineation, especially in lateral and anterior segments which normally are difficult to evaluate. In these segments more than half of the patients showed a better endocardial visibility. This effect was present at baseline examination as well as during peak dobutamine stress. In consequence, all other relevant aspects of dobutamine stress echocardiography, such as interobserver agreement and diagnostic accuracy, improved significantly using second harmonic imaging in comparison with conventional fundamental imaging.

The phenomenon of improved endocardial visibility owing to the use of second harmonic imaging is still not completely understood. Several factors contributing to improved endocardial delineation have been discussed. A recently published study showed that the grey value level of the endocardium is significantly increased using second harmonic imaging instead of fundamental imaging. ${ }^{27}$ The reduction of so called side lobe artefacts may result in a better near field quality and a reduction of clutter artefacts, ${ }^{28}$ which improved the endocardial delineation especially in the anterior and lateral segments. Harmonic signals that are generated by the penetrated tissue seem to be responsible for the better quality in the far field. ${ }^{10}{ }^{29}$ The higher receiver frequency compared to most conventionally used fundamental frequencies leads to an improved overall spatial resolution. 
The reduction of the number of segments with poor image quality is crucial for the feasibility of a stress echocardiography examination. This is shown by the fact that all non-diagnostic echocardiograms using conventional fundamental imaging became diagnostic using second harmonic imaging. Another advantage of second harmonic imaging in dobutamine stress echocardiography is its ease of use in clinical practice.

INTEROBSERVER AGREEMENT

Even though dobutamine stress echocardiography is an established clinical method, limited agreement between readers from different institutions in the interpretation of dobutamine stress echocardiography studies has been shown. ${ }^{7}$ The major parameter influencing inter-institutional agreement was shown to be the image quality of the dobutamine stress echocardiography study. While the majority agreement between five readers was found to be $100 \%$ for studies with higher image quality, it was only $45 \%$ for dobutamine stress echocardiography studies with poor image quality. These results indicated the need for the highest possible image quality to achieve a low heterogeneity in dobutamine stress echocardiography interpretation. Thus, with high image quality the insecurity in the interpretation of dobutamine stress echocardiography which may result in over interpretation as well as under interpretation is reduced. In the present study the $\kappa$ value as a parameter for the agreement between two independent observers increased from a moderate value of 0.40 using fundamental imaging to an excellent value of 0.69 with second harmonic imaging.

DIAGNOSTIC ACCURACY

Sensitivity and specificity of dobutamine stress echocardiography have been reported to be in the range of $54-96 \%$ and $66-97 \%$, respectively. ${ }^{1} 5617$ 30-32 The sensitivity of only $64 \%$ using conventional fundamental imaging in the present study is in the lower range of these previously reported data. This finding has to be explained by the selection of study patients with impaired native transthoracic image quality, but also by a high prevalence of pre-existing wall motion abnormalities, and the high number of patients with single vessel disease. ${ }^{4}$ This resulted in a sensitivity which was also significantly lower than commonly observed in our laboratory and reported for an unselected patient group. ${ }^{33}$

Using second harmonic imaging, the sensitivity and the accuracy of dobutamine stress echocardiography increased significantly to values in the higher range of previously published studies, which had been performed in patients with normal image quality. Improved accuracy was mainly caused by the fact that new or worsening wall motion abnormalities had been missed using conventional fundamental imaging technique in a relevant number of patients $(28 \%)$ and could only be detected using second harmonic imaging. Thus, in patients with impaired image quality a diagnostic accuracy could be obtained which had, until recently, been possible only in patients with a normal transthoracic image quality.

\section{STUDY LIMITATIONS}

A completely blinded interpretation of the dobutamine stress echocardiographic images was not possible in this study because second harmonic images can be identified by an experienced observer. The analysis of endocardial visibility scores might therefore be biased in favour of harmonic imaging. However, the fact that differences were found, particularly in the anterior and lateral walls, demonstrates a true benefit in image quality and not an overall difference in reading style. Furthermore, a possible bias should not be of great importance for the interpretation of wall motion abnormalities, because the two stress echocardiograms of a single patient (fundamental and harmonic) were evaluated separately and without knowledge of the patient's identity.

Patient inclusion criteria were confined to individuals with impaired image quality, which might bias the results in favour of harmonic imaging. The benefit of second harmonic imaging may have been smaller if patients with good or excellent image quality would have been included.

The fact that not all patients underwent coronary arteriography may possibly influence sensitivity and specificity as a post test referral bias. However, this influence remains equal for both stress echo modalities and does not diminish the benefit of harmonic imaging.

CONCLUSIONS AND FUTURE PERSPECTIVES

The present study demonstrated an improved endocardial visibility during dobutamine stress echocardiography using second harmonic compared with fundamental imaging, especially in the lateral and anterior segments, and an increased interobserver agreement. Moreover, the diagnostic accuracy of dobutamine stress echocardiography was improved using second harmonic imaging instead of conventional fundamental imaging in this selected group of patients with poor transthoracic echo window. Thus, second harmonic imaging allows the extension of the known high diagnostic accuracy of dobutamine stress echocardiography even to patients with impaired fundamental imaging quality. Studies using dobutamine stress echocardiography either for diagnosis of coronary artery disease or for the evaluation of the functional relevance of known coronary stenoses should use the beneficial effect of second harmonic imaging. Future studies will have to show the impact of second harmonic imaging on physical stress echocardiography as well as on inter-institutional agreement. The use of second harmonic imaging may improve the feasibility of automated border detection algorithms or three dimensional reconstruction at rest and during stress echocardiography. 
1 Sawada SG, Segar DS, Ryan T, et al. Echocardiographic detection of coronary artery disease during dobutamine

2 Picano E, Pingitore A, Conti U, et al. Enhanced sensitivity for detection of coronary artery disease by addition of atropine to dipyridamole echocardiography. Eur Heart $\mathcal{F} 1993$ 14:1216-22.

3 Picano E, Ostojic M, Varga A, et al. Combined low dose dipyridamole-dobutamine stress echocardiography to identify myocardial viability. $\mathcal{F}$ Am Coll Cardiol 1996;27:1422-8.

4 Bartunek J, Marwick TH, Rodrigues AC, et al. Dobutamineinduced wall motion abnormalities: correlations with myocardial fractional flow reserve and quantitative coronary angiography. 7 Am Coll Cardiol 1996;27:1429-36.

5 Cohen JL, Greene TO, Ottenweller J, et al. Dobutamine digital echocardiography for detecting coronary artery disease. Am f Cardiol 1991;67:1311-18.

6 Picano E, Lattanzi F, Orlandini A, et al. Stress echocardiography and the human factor: the importance of being expert. 7 Am Coll Cardiol 1991;17:666-9.

7 Hoffmann R, Lethen H, Marwick T, et al. Analysis of interinstitutional observer agreement in interpretation of dobutamine stres

8 Kornbluth M, Liang DH, Paloma A, et al. Native tissue harmonic imaging improves endocardial border definition and visualization of cardiac structures [abstract]. F $\mathrm{Am}$ Soc Echocardiogr 1998;11:693-701.

9 Spencer KT, Bednarz J, Rafter PG, et al. Use of harmonic imaging without echocardiographic contrast to improve two-dimensional image quality. Am f Cardiol 1998;82 794-9.

10 Becher H, Tiemann $\mathrm{K}$, Schlosser T, et al. Improvement in endocardial border delineation using tissue harmonic imaging. Echocardiography 1998;15:511-16.

11 de Jong N, Ten Cate FJ. New ultrasound contrast agents and technological innovations. Ultrasonics 1996;34:587-90.

12 Burns PN. Harmonic imaging with ultrasound contrast agents. Clin Radiol 1996;51 (suppl 1):50-5.

13 Schiller NB, Shah PM, Crawford M, et al. Recommendations for quantitation of the left ventricle by twodimensional echocardiography. American Society of Echodimensional echocardiography. American Society of Echocardiography committee on standards, subcommittee on
quantitation of two-dimensional echocardiograms. I $\mathrm{Am}$ Soc Echocardiogr 1989;2:358-67.

14 Gibbons RJ, Balady GJ, Beasley JW, et al. ACC/AHA guidelines for exercise testing: executive summary. A report of the American College of Cardiology/American Heart Association task force on practice guidelines (committee on exercise testing). Circulation 1997;96:345-54

15 McNeill AJ, Fioretti PM, el-Said SM, et al. Enhanced sensitivity for detection of coronary artery disease by addition of atropine to dobutamine stress echocardiography. $A m$ of Cardiol 1992;70:41-6.

16 Cohen J. A coefficient of agreement for nominal scales. Educ Psychol Measurements 1960;20:37-46.

17 Marwick TH, Nemec JJ, Pashkow FJ, et al. Accuracy and limitations of exercise echocardiography in a routine clinical setting. $7 \mathrm{Am}$ Coll Cardiol 1992;19:74-81.

18 Mor AV, Vignon P, Koch R, et al. Segmental analysis of color kinesis images: new method for quantification of the magnitude and timing of endocardial motion during left ventricular systole and diastole. Circulation 1997;95:208297.
19 Katz WE, Gulati VK, Mahler CM, et al. Quantitative evaluation of the segmental left ventricular response to dobutamine stress by tissue Doppler echocardiography. $\mathrm{Am}$ f Cardiol 1997;79:1036-42.

20 Falcone RA, Marcovitz PA, Perez JE, et al. Intravenous albunex during dobutamine stress echocardiography: enhanced localization of left ventricular endocardial borders. Am Heart f 1995;130:254-8.

21 Leischik R, Kuhlmann C, Bruch C, et al. Reproducibility of stress echocardiography using intravenous injection of ultrasound contrast agent (BY 963). Int $\mathcal{f}$ Card Imaging 1997;13:387-94.

22 Porter TR, Xie F, Kricsfeld A, et al. Improved endocardial border resolution during dobutamine stress echocardiography with intravenous sonicated dextrose albumin. $7 \mathrm{Am}$ Coll Cardiol 1994;23:1440-3.

23 Agati L, Renzi M, Sciomer S, et al. Transesophageal dipyridamole echocardiography for diagnosis of coronary artery disease. $7 \mathrm{Am}$ Coll Cardiol 1992;19:765-70

24 Flachskampf FA, Lethen H, Hoffmann R, et al. Transoesophageal stress echocardiography. Eur Heart f 1997; 18(suppl D):D37-D42.

25 Lambertz H, Kreis A, Trümper $\mathrm{H}$, et al. Simultaneous transesophageal atrial pacing and transesophageal twodimensional echocardiography: a new method of stress echocardiography. 7 Am Coll Cardiol 1990;16:1143-53.

26 Mulvagh SL, Foley DA, Gilman GM, et al. Noncontrast tissue harmonic imaging markedly enhances image quality in technically difficult echocardiograms [abstract]. $\mathcal{F}$ Am Coll Cardiol 1998;31:76A.

27 Caidahl K, Kazzam E, Lidberg J, et al. New concepts in echocardiography: harmonic imaging of tissue without use of contrast agents. Lancet 1998;352:1264-70.

28 Rubin DN, Yazbek N, Homa D, et al. Why does tissue harmonic imaging improve image quality: a quantitative examination demonstrating side-lobe suppression [abstract]. F Am Coll Cardiol 1998;31:127A.

29 Averkiou MA, Roundhill DN, Powers JE. A new imaging technique based on the nonlinear properties of tissues. In: Schneider SC, Levy M, AcAvoy, eds. IEEE Ultrasonics Symposium. Toronto: Institute of Electrical and Electronic Enmpineers, 1997;2:1561-6.

30 Marcovitz PA, Armstrong WF. Accuracy of dobutamine stress echocardiography in detecting coronary artery disease. Am f Cardiol 1992;69:1269-73.

31 Mairesse GH, Marwick TH, Vanoverschelde JL, et al. How accurate is dobutamine stress electrocardiography for detection of coronary artery disease? Comparison with two-dimensional echocardiography and technetium-99m methoxyl isobutyl isonitrile (mibi) perfusion scintigraphy. $\mathcal{F}$ Am Coll Cardiol 1994:24:920-7.

32 Geleijnse ML, Salustri A, Marwick TH, et al. Should the diagnosis of coronary artery disease be based on the evaluation of myocardial function or perfusion? Eur Heart $\mathcal{F}$ 1997;18(suppl D):D68-77.

33 Hoffmann R, Lethen H, Kleinhans E, et al. Comparative evaluation of bicycle and dobutamine stress echocardiography with perfusion scintigraphy and bicycle electrocardiogram for identification of coronary artery disease. Am $\mathcal{F}$ Cardiol 1993;72:555-9. 